\begin{tabular}{|c|l|}
\hline Title & Properties of electron swarms in gases in the upstream region of an electron source \\
\hline Author(s) & Sugawara, H.; Sakai, Y.; T agashira, H. \\
\hline Citation & Journal of Physics D: A pplied Physics, 28(1), 61-67 \\
\hline https://doi.org/10.1088/0022-3727/28/1/11
\end{tabular}

Instructions for use 


\title{
Properties of electron swarms in gases in the upstream region of an electron source $z$
}

\author{
H Sugawara, Y Sakai and H Tagashira \\ Department of Electrical Engineering, Hokkaido University, Sapporo $060 \mathrm{~J}$ apan
}

\begin{abstract}
A bstract. Exponential spatial growth of electron swarms in gases under steady-state Townsend conditions may be observed not only in the downstream region of an electron source but also in the upstream region due to backward diãusion. Relations among swarm parameters in the upstream region were deduced and found to have peculiar characteristics. For example, the sign of the average electron velocity $v_{d}$ for steadystate Townsend conditions depends on whether the gas is electro-positive or electronegative. This property is conårmed quantitatively by calculating the electron energy distribution using a propagator method modiåed for analysis in the upstream region. As an example of the eãect of backward diãusion, the decay in the electron number density in front of the anode in a steady-state Townsend experiment between parallel plane electrodes was studied. The decay is caused by the missing electrons absorbed at the anode. The energy distribution of the missing electrons has a non-equilibrium relaxation region and the equilibrium region lies towards the cathode direction. The number density of the missing electrons showed an exponential spatial dependence in the equilibrium region. The relative gradient of the decrease agreed well with the relative density gradient ã obtained in the upstream region. It was also found that the electron energy distribution in the decaying region tends to that in the upstream equilibrium region.
\end{abstract}

PACS numbers: $52.80 .-\mathrm{s}$ Electric discharges

\section{Introduction}

A steady-state electron swarm is formed by continuously supplying initial electrons from a point source in gases in the presence of an electric åeld. As shown in ågure 1, three regions can be deåned relative to the source location; the near-source region, the downstream region (DSR) and the upstream region (USR). The near-source region is a region of non-equilibrium relaxation. The DSR and USR are regions in equilibrium.

z Published source: J ournal of Physics D: A pplied Physics, Vol. 28 (1995), pp. 61-67. 
Here, we deåned downstream as being the direction in which an electron would move in the presence of an electric åeld, E, in a collisionless environment. The USR is simply the opposite direction. In the DSR, as the distance from the source increases, the electron number density exponentially increases by ionization or decreases by attachment. This is the well-known steady-state Townsend (SST) condition. The assumption of an exponentially growing electron number density with distance from the source is the basis for analysis of electron swarms to obtain the eãective ionization coeé cient ã.

B ecause of collisions, electrons can be found not only in the DSR but also in the USR as has been demonstrated for example by a M onte Carlo simulation (MCS) (B raglia and Lowke, 1979). Here, we deåne backward diãusion as being the mechanism by which electrons penetrate the USR. Although most of the literature has dealt with the DSR, a number of investigators have addressed electron behaviour in the USR.

Tagashira $(1985,1991)$ suggested that the two solutions for ã, obtained by solving the continuity equation assuming exponentially varying electron number densities in space, correspond to the coee cients of the relative density gradient in the USR and DSR. Standish (1989) solved a kinetic equation for the steady-state spatial distributions of charged particles using eigenfunctions for particle transport. His analysis showed the exponential spatial dependence of the electron density in both the USR and DSR. The conclusion that the two solutions for ã are the real roots of the zeroth-order dispersion relation for electron transport and that they correspond to the USR and DSR was obtained by Standish (1989), K ondo and Tagashira (1990) and Robson (1991).

Chantry (1982) investigated the decay in the electron number density in front of an absorbing anode in terms of backward-diãusing electrons. It is shown in the present paper that the behaviour of backward-diãusing vacancies, namely missing electrons due to the absorption, may be equivalent to that of electron swarms in the USR .

$K$ nowing the characteristics of backward-diãusing electron swarms may permit one to extend the treatment of the properties of electron swarms. Therefore, in order to develop the theory of electron swarms further, analyses for backward-diãusing electrons in the USR as well as those for the DSR are important.

Characteristics of the electron energy distribution and relations among the electron swarm parameters in the USR are discussed in section 2. In order to conarrm the relations, the electron energy distribution in the USR is calculated in section 3 under a uniform electric åeld using a previously developed propagator method (PM) (Sugawara et al 1994) but implemented here for the USR. A discussion of results is given in section 4. The problem of the decay in the electron number density in front of the anode of parallel plane electrodes is an example of application of the principle and technique investigated in the present paper. This problem is treated in section 5, where a comparison is made between results by the PM and a MCS to prove the validity of application of the present PM to the problem. 


\section{Relations among swarm parameters}

\subsection{The relative density gradient coeé cient}

The continuity equation for electrons under SST conditions may be described using SST swarm parameters (T homas 1969, Tagashira et al 1977, B levin and Fletcher 1984). Following B levin and Fletcher (1984), when an exponential solution $n_{e}(x)=n_{0} \exp (a ̃ x)$ for the electron number density at position $x$ is assumed for an electron source located at $x=0$, the continuity equation is described using the eãective ionization frequency $R_{i s}$, the drift velocity $W_{s}$ and the diãusion coeé cient $D_{s}$ for SST conditions as

$$
R_{\text {is }} n_{e}(x) \ddot{A} W_{s} \frac{@}{@ x} n_{e}(x)+D_{s} \frac{@}{@ x^{2}} n_{e}(x)=0:
$$

These SST parameters were deåned by rearranging all the higher order parameters for the time-of-çight (TOF) experiment. Since the spatial growth is assumed to be exponential, we may replace the operator @-@ with ã to give

$$
R_{\text {is }} n_{0} \ddot{A} \text { ã } W_{s} n_{0}+\tilde{a}^{2} D_{s} n_{0}=0:
$$

T wo solutions for ã are obtained as follows.

$$
\tilde{a}=\frac{W_{s} \ddot{U}{ }^{q} \overline{W_{s}^{2} \ddot{A} 4 R_{\text {is }} D_{s}}}{2 D_{s}}
$$

Tagashira (1985) inferred that these solutions correspond respectively to the USR $(x<0)$ and the DSR $(x>0)$. For the DSR, the negative sign is adopted for ã so that ã becomes zero when $R_{\text {is }}$ tends to zero.

In contrast, ã $x$ in the USR should always have a negative value so that the electron number density al ways decays in the upstream direction irrespective of the sign of $R_{\text {is. }}$. In this case, ã simply represents the relative gradient of the electron number density.

As shown by Standish (1989), Kondo and Tagashira (1990) and Robson (1991), there are two diãerent eigenvalues for the values of ã to represent exponential growth in the USR and DSR. The equilibrium electron energy distributions for the USR and DSR should be diãerent from each other since they are determined by diã erent eigenvalues or relative density gradients. Therefore, the electron energy distributions are diãerent and swarm parameters such as $W_{S}, D_{s}$ and $R_{\text {is }}$ should have diãerent values for the USR and DSR, although the formulations to deduce them from the energy distributions should be identical for both USR and DSR (Tagashira et al , 1977, section 3.3; Blevin and Fletcher, 1984, section 3(b)).

Hereafter, the primed swarm parameters indicate those obtained from the energy distribution in the USR while the unprimed ones indicate those of the DSR as follows.

$$
\tilde{a}=\frac{W_{s} \ddot{A}^{q} \overline{W_{s}^{2} \ddot{A} 4 R_{i s} D_{s}}}{2 D_{s}} \quad \text { for the downstream region }
$$




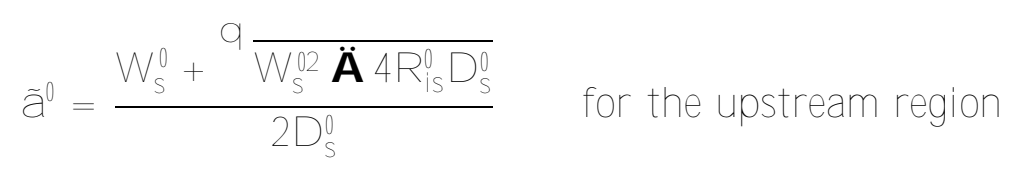

It is important to note that the reason for using diãerent values of the drift velocity, diãusion coeé cient and ionization frequency in the USR and DSR comes from the fact that the SST swarm parameters are dependent on the electron concentration gradient and are quite diãerent from the concentration-gradient-independent TOF parameters (Tagashira et al 1977) in this respect.

\subsection{Drift velocity}

If an exponential distribution, $n_{0} \exp \left(a^{a} x\right)$, is assumed for the electrons in the USR (Standish, 1989), then the total number of electrons, $\mathrm{N}_{\mathrm{e}}(\times 9)$, on the upstream side of $\left.x=x^{9}<0\right)$ has a ånite value and can be written as

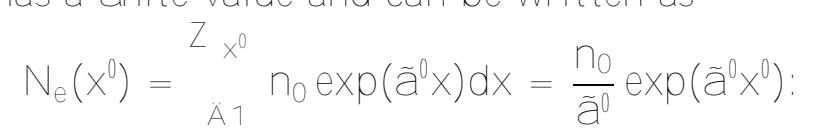

Since $N_{e}(x)$ is constant under a steady state, the electron generation per unit time $\mathrm{R}_{\text {is }}^{0} \mathrm{~N}_{\mathrm{e}}\left(\mathrm{x} 9\right.$ in this region must be equal to the electron outç ow across $\mathrm{x}=\mathrm{x}^{0}$ towards the DSR per unit time. Equating the outçow, which is the çux denoted as $n_{0} \exp (\tilde{a} q x) v_{d}^{0}$, to the generation rate, one obtains

$$
\mathrm{R}_{\text {is }}^{0} \frac{\mathrm{n}_{0}}{\tilde{a} 0} \exp \left(\tilde{a} q_{x} g=n_{0} \exp (\tilde{a} q) q v_{d}^{0}\right. \text { : }
$$

Here, $v_{d}^{0}$ is the SST average velocity (ë çux/density, Robson, 1991), which is identical to the diãusion-modiåed drift velocity (ë $W_{s}^{0} \ddot{A} a ̃ D_{s}^{0}$, Tagashira et al , 1977) under SST conditions. This velocity represents the average of the velocity component parallel to $E$ for SST conditions. Equation (7) can be simpliåed to

$$
R_{\text {is }}^{0}=v_{d}^{0} \tilde{a}^{0}
$$

which is similar to $R_{\text {is }}=v_{d} a$ in the DSR. An equivalent discussion may be made by using a diãerential form of the continuity equation of electrons for SST .

Since $v_{d}>0$ is always satisåed in the DSR, the same sign is shared by $R_{\text {is }}$ and ã. The relative gradient, ã, of $n_{e}(x)=n_{0} \exp (a \tilde{x})$ directly represents electron multiplication by ionization $\left(R_{\text {is }}>0\right)$ or decay by electron attachment $\left(R_{\text {is }}<0\right)$. On the other hand, since $a^{0}$ in the USR always has a positive value, $R_{\text {is }}^{0}$ and $v_{d}^{0}$ should share the same sign. Therefore, the sign of average velocity $v_{d}^{0}$, as well as that of $R_{\text {is }}^{0}$ indicates whether the gas is eãectively electropositive or electronegative.

In the special case that the number of electrons is conserved, or the gain of electrons by ionization exactly balances the loss by attachment, $v_{d}^{0}$ becomes zero since $R_{i s}^{0}=0$. Under this condition, the relation $W_{s}^{0}=\tilde{a} D_{s}^{0}$, deduced from equation (2), represents a balance between drift by the electric åeld and backward diãusion as a result of the density gradient. 


\section{Calculation of the electron energy distribution}

In the preceding section, it was shown that the relation among swarm parameters in the USR is exactly analogous to that in the DSR. Next, the validity of equations (5) and (8) is demonstrated by a simulation using a propagator method which was previously used by the authors for analysis in the DSR but is modiåed here for analysis in the USR.

\subsection{Numerical method for the upstream region}

A numerical technique based on a propagator method (Sugawara et al 1994) for the calculation of equilibrium energy distribution in the DSR under SST conditions was modiåed for the USR. In the propagator method, the electron energy distribution $F\left(e_{i} i\right)$ is represented as a function of the electron energy è and the angle í between $\mathrm{E}$ and the direction of electron motion. Phase space (è $i$ ) is devided into many small cells for every $\AA$ è and $\AA$ í . For every time step, electron propagation among the cells is calculated using operators called propagators, which represent the acceleration due to $\mathrm{E}$ and scattering by collisions with gas molecules.

In the implementation of the previous PM, the assumption of exponential spatial growth in the DSR enabled us to obtain equilibrium solutions for $F\left(e_{i} ; I^{\prime}\right.$ in a thin slab normal to the electron çow without considering the spatial relaxation process. The PM is now applied for analyses in the USR, since the formulation of the continuity equation in the USR is analogous to that for the DSR.

U sing a similar technique to the previous PM, the quantity $\exp (\tilde{a} \cap \mathrm{x})$ is obtained from the following conservation equation by describing the variation of the number of electrons in a thin slab with a thickness $\AA \mathrm{x}$;

$$
n_{b} f \exp (a ̃ \AA x) \ddot{A} 1 g+n_{f} f \exp (\ddot{A} a ̃ \AA x) \ddot{A} 1 g+\left(n_{i} \ddot{A} n_{a}\right)=0:
$$

Here, $n_{f}, n_{b}, n_{i}$ and $n_{a}$ are the changes of the number of electrons in a time step corresponding to respectively forward and backward outçows, ionization and attachment. Equation (9) has two solutions;

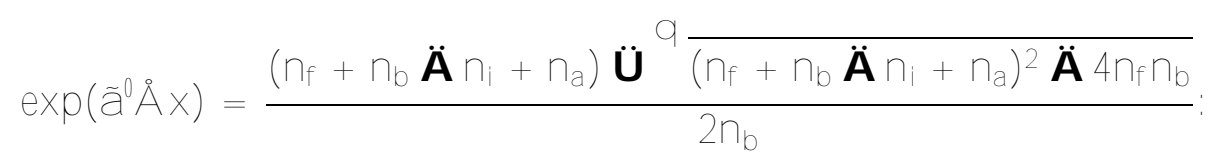

The sign of the solution is chosen in the same way as discussed in section 2.1; the positive sign is adopted for $\tilde{a}^{0}$, since it always has a positive value in the USR. Note that the choice of the negative sign was shown to be valid for determining the electron energy distributions and swarm parameters in the DSR in the previous PM (Sugawara et al 1994). 
Here, using a Taylor expansion as $\exp (\ddot{A} a ̃ o \AA x) ~ ' 1 \ddot{A}$ ã $\AA$ x , equation (10) becomes $\tilde{a}^{0}=\frac{1}{\AA x} \AA^{\left(n_{f} \ddot{A} n_{b}+n_{i} \ddot{A} n_{a}\right)+{ }^{q} \frac{\left(n_{f} \ddot{A} n_{b}+n_{i} \ddot{A} n_{a}\right)^{2} \ddot{A} 4 n_{f}\left(n_{i} \ddot{A} n_{a}\right)}{2 n_{f}}:}$

The exact value of $a^{0}$ should be obtained when $\AA x$ tends to zero. Although $\AA x$ in practical calculations has a non-zero value, $\AA \mathrm{x}$ is set to be as small as possible in the calculation.

The calculation is started using an appropriate choice of the initial distribution of $F(e ̀ i)$ and is repeated until the distribution reaches equilibrium. In the USR, some of the electrons must have sue ciently high energies that they can diãuse backwards against $E$. Therefore, a high enough maximum energy èmax and appropriate values for $F$ (èmax $; i ́$ ) were chosen for calculation of $F(\grave{e} ; i)$. To conårm the validity of the solutions, an energy balance (T homas 1969 equation (9)) was performed to demonstrate the drift equiliblium here in the USR.

\subsection{Gases and electric ådds}

Three classes of gases were chosen to conårm equation (8); argon as an electro-positive gas (case $1, R_{i s}^{0}>0$ ), a ramp model gas in which ionization and attachment do not occur (case $2, R_{i s}^{0}=0$ ), and sulphur hexaçuoride as an electronegative gas (case $3, R_{i s}^{0}<0$ ). The sets of cross sections used in the present work are based on those of Suzuki et al (1990), M ason and Newell (1987) and Sakai et al (1972) for argon, Reid (1979) for the ramp model gas and Itoh et al (1993) for sulphur hexaçuoride.

The values of the reduced electric åeld are chosen to be $E=N=1410,283$ and $141 \mathrm{Td}$ for cases 1, 2 and 3 respectively. For case 1 , a high $E=\mathrm{N}$ is chosen so that $R_{\text {is }}^{0}$ is large enough to give a signiåcant value of $\mathrm{v}_{\mathrm{d}}^{0}$. On the other hand, a low $\mathrm{E}=\mathrm{N}$ is chosen for case 3 so that $R_{\text {is }}^{0}$ is a large negative quantity. These are chosen to emphasize the ionization in case 1 and attachment in case 3 . The number density of gas molecules, $\mathrm{N}$, is assumed to be $3: 53$ Ç $10^{16} \mathrm{~cm}^{\ddot{A} 3}$ ( 1 Torr at $0^{\circ} \mathrm{C}$ ).

\section{Results and discussion}

\subsection{The electron energy distribution}

$F_{0}(e)$, the isotropic part of $F\left(e_{i} i\right), F_{1}(e ̀)$, an anisotropic part, and all other terms of the Legendre expansion can be obtained from the exact solution $F\left(e_{i} i\right.$ ) calculated here by the $\mathrm{PM} . \mathrm{F}_{0}(\mathrm{e})$ and $\mathrm{F}_{1}(\mathrm{e})$ are shown in ågure 2. There are two important diãerences between the electron energy distributions in the USR and the DSR. In contrast to those in the DSR, the electron energies in the USR are in general lower and on the average the high-energy electrons move backwards. 
Electrons diãuse backwards into the USR because of the large gradient in the electron number density. In this case, only high-energy electrons can diãuse backwards since diãusion against $E$ results in rapid energy loss. Some electrons lose part of their energy by collisions during their stay in $x<x^{0}$, where $x^{0}$ is a location in the USR. When they eventually lose enough energy, they drift towards the DSR unless they are lost in the USR by attachment. If super-elastic collisions are neglected, then those electrons that have undergone backward diãusion will necessarily have a lower energy at $x^{0}$ than those that have not undergone backward diãusion. Therefore, $F_{1}(e ̀)$ will be positive for low energy electrons as shown in ågures $2(\mathrm{a})$ and (c). In the electronegative gas, the number of electrons decreases by attachment as they diãuse backwards. As a result, $\mathrm{F}_{1}(\mathrm{e})$ is negative for all è for the conditions in ågure $2(\mathrm{e})$.

As expected, a shift in $\mathrm{F}_{0}$ (è) towards higher energies with increasing $E=\mathrm{N}$ was observed even in the USR. This is similar to what occurs in the DSR. A high $E=N$ prevents low energy electrons from diãusing backwards. The total number of electrons penetrating into the USR, as denoted in equation (6), decreases since ã 0 increases with increasing $E=N$. An exponential spatial distribution at a high $E=N$ is formed by backward diãusion of electrons with commensurately larger energies.

\subsection{Electron swarm parameters}

The trends in the energy distribution presented in ågure 2 signiåcantly aãect the swarm parameters. The mean energy $\tilde{e}$, the eãective ionization frequency $R_{i s^{\prime}}^{0}$ the relative gradient of the electron number density $\tilde{a}^{0}$, the quantity $R_{\text {is }}^{0}=\tilde{a}^{0}$ and the drift velocity $v_{d}^{0}$ in the USR are listed in table 1 together with those in the DSR under the same $E=N$ for comparison. A comparison between $v_{d}^{0}$ and $R_{\text {is }}^{0}=a^{0}$ conårms equation (8). Here, $v_{d}^{0}$ is given as $\frac{1}{3} \mathrm{R}_{1} \quad V_{1} \mathrm{P}_{\mathrm{e}_{\mathrm{F}}}$ (è)dè where $\mathrm{V}_{1}$ is the speed of an electron with the energy of $1 \mathrm{eV}$. The values of $R_{i s}^{0}=\tilde{a}^{0}$ and $v_{d}^{0}$ are found to agree well with each other to within a fraction of a percent.

Table 1. List of swarm parameters.

\begin{tabular}{|c|c|c|c|c|c|c|c|}
\hline gas & region & $E=N$ & $\begin{array}{c}\tilde{\mathrm{e}} \\
\tilde{\mathrm{\theta}} \\
(\mathrm{eV})\end{array}$ & $\begin{array}{c}R_{\text {is }}^{0} \\
R_{\text {is }} \\
\left(\tilde{n} s^{\ddot{A} 1}\right)\end{array}$ & $\begin{array}{c}\tilde{a}^{0} \\
\tilde{a} \\
\left(\mathrm{~cm}^{\ddot{A} 1}\right)\end{array}$ & $\begin{array}{c}\mathrm{R}_{\text {is }}^{0}=\tilde{a}{ }^{0} \\
R_{\text {is }}=\tilde{a} \\
(\mathrm{~cm} / \tilde{n} \mathrm{~s})\end{array}$ & $\begin{array}{c}v_{d}^{0} \\
v_{d} \\
(\mathrm{~cm} / \tilde{n} \mathrm{~s})\end{array}$ \\
\hline $\mathrm{Ar}$ & upstream & 1410 & 5.37 & 17.3 & 138 & 0.126 & 0.125 \\
\hline (case (1)) & downstream & 1410 & 15.7 & 590 & 10.1 & 58.2 & 57.9 \\
\hline ramp model gas & upstream & 283 & 0.54 & 0.0 & 270 & 0.0 & -0.004 \\
\hline (case (2)) & downstream & 283 & 2.13 & 0.0 & 0.0 & |* & 13.3 \\
\hline $\mathrm{SF}_{6}$ & upstream & 141 & 2.71 & -112 & 33.6 & -3.33 & -3.30 \\
\hline (case (3)) & downstream & 141 & 5.62 & -30.1 & -2.4 & 12.3 & 12.3 \\
\hline
\end{tabular}


Typically, an electron swarm under SST conditions can be regarded as the integral of an isolated swarm with respect to time at a åxed position (Sakai et al , 1977). In section 2.2, the fact that $v_{d}^{0}=0$ when $R_{\text {is }}^{0}=0$ is explained using a macroscopic approach. This conclusion can also be attained with a microscopic approach, that is by looking at the behaviour of an individual electron. Following Sakai et al (1977), the contribution of an individual electron to the drift velocity $v_{d}^{0}$ is the electron velocity component parallel to $E$ weighted by its residence time in a small interval in space with a thickness $\AA \mathrm{x}$. If there is no production and loss of electrons, an electron that passes across $\AA \mathrm{x}$ in the USR must return across $\AA x$ towards the DSR, hence the number of crossings by an individual electron across $\AA \mathrm{x}$ is necessarily even. Since the residence time is inversely proportional to the velocity component parallel to $\mathrm{E}$ and the velocity changes sign with each crossing, the net contribution to the drift velocity is zero. In contrast, the number of crossings is odd in the DSR. The diãerence in the parity of the number of crossings in the two regions is the key diã erence between the drift velocities for the USR and DSR.

\section{A practical example of backward diãusion}

\subsection{The inçuence of absorbing anode}

A practical example of backward diãusion in the USR, as previously discussed, is seen in an SST experiment between parallel plane electrodes. The decay in the electron number density in front of an absorbing anode may be regarded as the appearence of vacancies due to the absorption of electrons at the anode. Here, we deane the vacancy as being missing electrons.

Chantry (1982) discussed the eãect of an absorbing anode on the SST electron swarm using a concept of complementarity. The complementarity theorem of Chantry (1982) is as follows. If a plane which is perpendicular to $E$ is placed in the region where the electron swarm is in equilibrium, electrons near the plane are a mixture of those electrons that are diãusing forwards and those that are diãusing backwards across the plane. When we regard the plane as an absorbing anode, swarm parameters on the upstream side near the plane would vary spatially corresponding to the contribution of backward-diãusing electrons to the energy distribution, since these electrons would be lost by absorption.

If those electrons absorbed at the anode could move freely as if they were in the presence of the electric åeld even after absorption, then they could diãuse backwards and åll the vacancy. Here, the energy distribution of backward-diãusing electrons $F_{v}(e ̀ r)$ necessary to åll the vacancies is deåned as

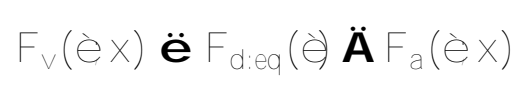

where $F_{d: e q}(e)$ and $F_{a}(e ̀ ; x)$ are the electron energy distributions at positions in the 
equilibrium region in the DSR and vicinity of the anode respectively as shown in ågure 3 .

\subsection{Monte Carlo simulation}

To test the concept of backward diãusion of vacancies as deåned by equation (12), a M onte Carlo simulation (MCS) was performed to obtain $F_{v}(e ̀ ; x)$. It is assumed that the initial electrons are supplied at a plane source and they drift and diãuse downstream. Those electrons which arrive at the position of an imaginary anode are labelled to indicate that they are absorbed. They are then allowed to continue propagating under the inçuence of the electric åeld. The electrons that return across the anode are sampled yielding $F_{v}\left(e_{i} ; x\right)$ of electrons which diãuse backwards from the anode. In this case, the anode becomes equivalent to a source; the region in front of the anode becomes the USR; the region 'inside' the anode becomes the DSR .

A s a benchmark case, a simulation was performed for a ramp model gas. The initial electrons were given a uniform energy distribution between 0 and $10 \mathrm{eV}$. The distance from the electron source to the anode was $5 \mathrm{~mm}$. This distance is long enough for the electrons to reach equilibrium under the same conditions as those given in section 3.2.

The results from the MCS are shown in ågures 4 and 5 . In ågure 4 , the energy distributions of the vacancies, $F_{v}(e ; x)$, are presented as a function of the distance from the anode. An important feature to note is that the shape of $F_{v}\left(e_{i} ; x\right)$ converges to $F_{\text {u:eq }}(\hat{e})$, where $F_{u: e q}(\grave{e})$ is the equilibrium electron energy distribution in the USR. In the region in ågure 5 , where the gradient of the logarithm of the electron number density agrees with $a^{0}$ in table 1 , the shape of $F_{v}\left(e_{i} x\right)$ in ågure 4 is essentially the same as $F_{u: e q}(e ̀)$. This shows that the backward-diãusing electrons from the anode also undergo processes of relaxation and exponential decay essentially similar to the behaviour of the backward-diãusing electrons in the USR .

B ecause of the large value of $\tilde{a}^{0}$, there is a steep decay in the number density of backward-diãusing electrons adjacent to the anode. The resulting low number density of these electrons at increasing distances from the anode $(<A \ddot{0} 0: 05 \mathrm{~cm})$ results in the statistical çuctuations observed in ågure 5 .

\section{Conclusions}

The properties of electron swarms in gases in the upstream region of the electron source, formed by backward diãusion of electrons against the electric åeld, are studied under steady-state Townsend conditions.

Relations among electron swarm parameters for the upstream region are deduced under the assumption of exponential spatial growth. It is shown that the sign of the electron drift velocity $v_{d}$ is positive when the gas is electropositive, and $v_{d}$ is negative when the gas is electronegative. $v_{d}$ is zero where there is no ionization or attachment. 
These characteristics are conårmed by calculating the electron energy distribution using a previously developed propagator method (Sugawara et al 1994) but modiåed here for analyses in the upstream region.

A steady-state electron swarm between parallel plane electrodes is simulated using a $\mathrm{M}$ onte $\mathrm{C}$ arlo method to show that the decay in the electron number density in front of the absorbing anode is due to vacancies created by electrons absorbed at the anode. The exponential decay in the electron number density with distance was also conårmed by the $\mathrm{M}$ onte $\mathrm{C}$ arlo simulation. The electron energy distribution when the decay reaches equilibrium and becomes exponential is demonstrated to be the equilibrium electron energy distribution in the USR.

The equilibrium behaviour of these electrons is shown to be essentially the same as that in the upstream region. The agreement between the electron energy distributions calculated by the propagator methods and the Monte Carlo simulation validates the use of the present propagator method to predict electron swarm behaviour in the upstream region.

\section{A cknowledgment}

The authors wish to thank P rofessor $\mathrm{K}$ K ondo of A nan Technical College and Professor P L G Ventzek, Drs H Date and Y Ohmori, their laboratory colleagues, for stimulating suggestions and discussions.

\section{R eferences}

Blevin H A and Fletcher J 1984 Aust. J. Phys. $37593\{600$

Braglia G L and Lowke J J 1979 J. Phys. D: A ppl. Phys. $121831\{8$

Chantry P J 1982 Phys. Rev. A 25 1209\{13

Itoh H, Miura Y, I kuta N, Nakao Y and Tagashira H 1988 J. Phys. D: A ppl. P hys. $21922\{30$

Itoh H, Matsumura T, Satoh K, Date H, Nakao Y and Tagashira H $1993 \mathrm{~J}$. Phys. D: Appl. Phys. 26 $1975\{9$

K ondo K and Tagashira H 1990 J. Phys. D: A ppl. Phys. $231175\{83$

Mason N J and Newell W R 1987 J. Phys. B: At. Mol. Phys. $51357\{77$

Reid I D 1979 Aust. J. Phys. $32231\{54$

Robson R E 1991 Aust. J. Phys. $44685\{92$

| | 1991 Gaseous Electronics and Its Applications, Ed. by Crompton R W et al , KTK Scientiåc Publishers (Tokyo) $89\{101$

Sakai Y, Tagashira H and Sakamoto S 1972 J . Phys. B: At. Mol. Phys. $51010\{6$

| | 1977 J. Phys. D: A ppl. Phys. $101035\{49$

Standish R K 1989 Aust. J. Phys. $42223\{32$

Sugawara H, Sakai $Y$ and Tagashira H 1994 J. Phys. D: A ppl. Phys. $2790\{4$

Suzuki M, Taniguchi T and Tagashira H 1990 J. P hys. D: Appl. Phys. $23842\{50$

Tagashira H, Sakai Y and Sakamoto S 1977 J. Phys. D: A ppl. P hys. $101051\{63$ 
Tagashira H 1985 Papars of Technical Committee for Electrical Discharges, IE E J apan ED-85-115 | | 1991 Trans. IEE J apan 111-A 147\{51

Thomas W R L 1969 J. Phys. B: At. Mol. Phys. $2551\{61$ 


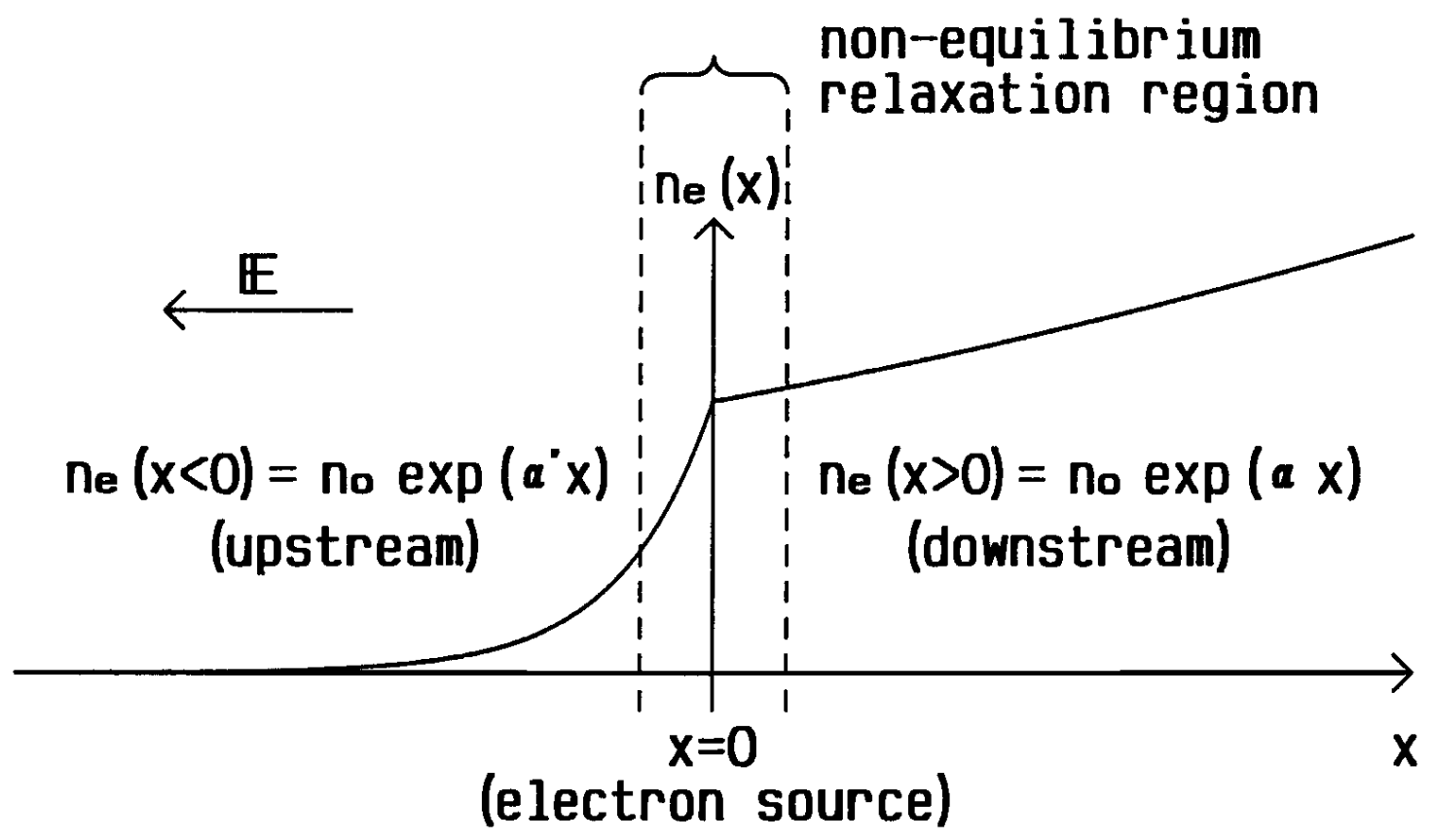

Figure 1. The model of steady-state electron flow on each side of the electron source. 


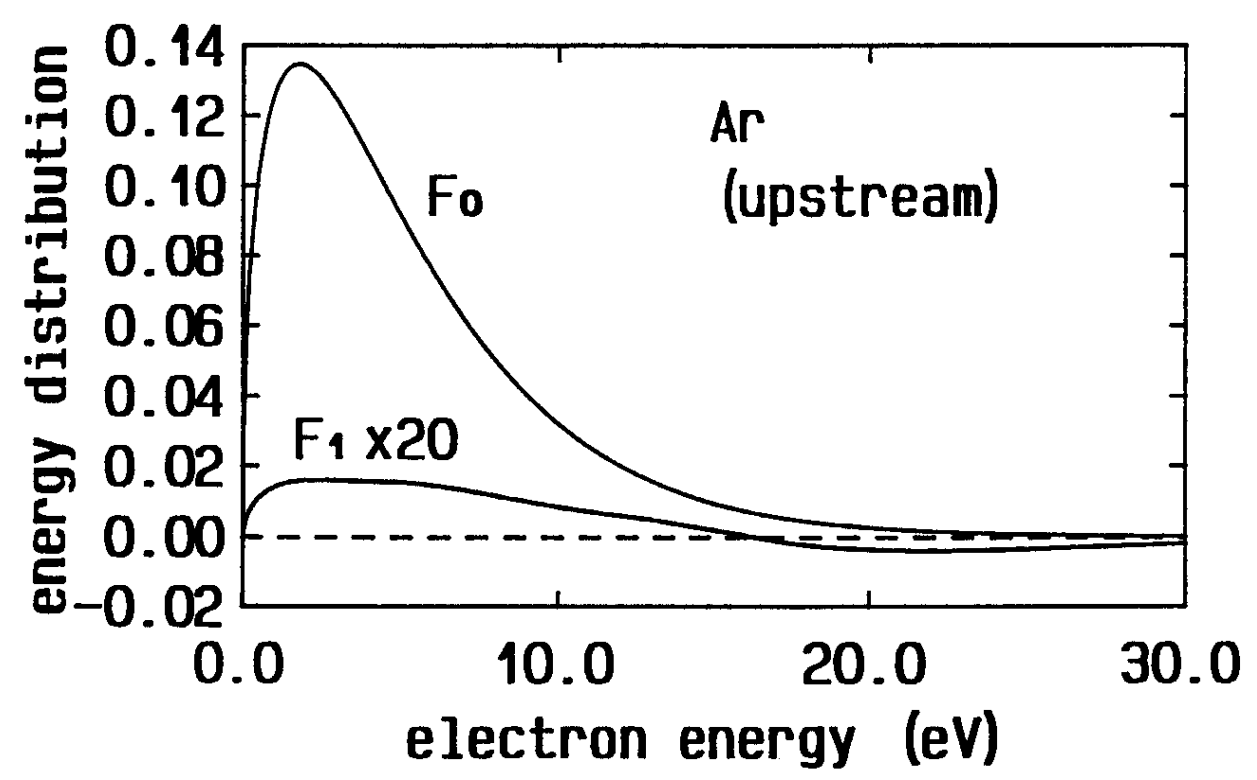

Figure 2(a)

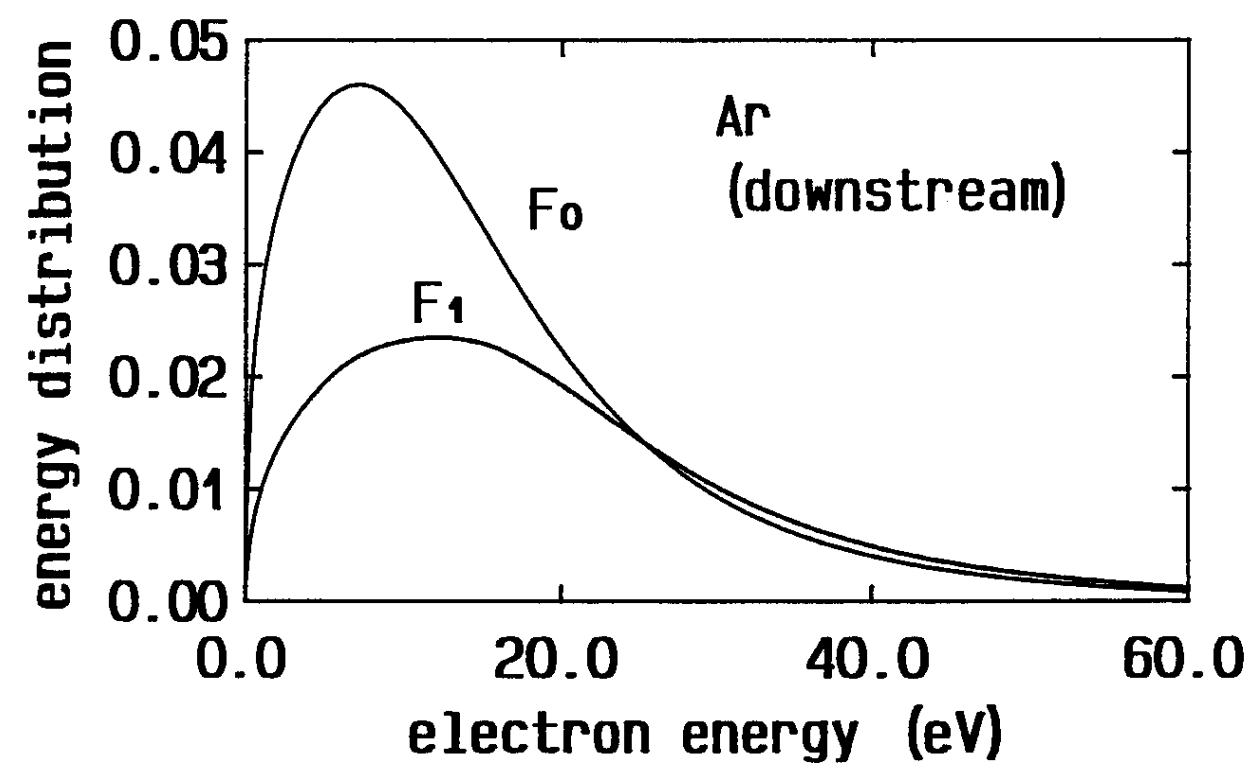

Figure 2(b)

Figure 2. Electron energy distributions calculated using a propagator technique: (a) and (b), argon at $E / N=$ $1410 \mathrm{Td}$; (c) and (d), ramp model gas at $E / N=283 \mathrm{Td}$; (e) and (f), sulphur hexafluoride at $E / N=141 \mathrm{Td}$. Figures (a), (c) and (e) are for the upstream region, while figures (b), (d) and (f) are for the downstream region. 


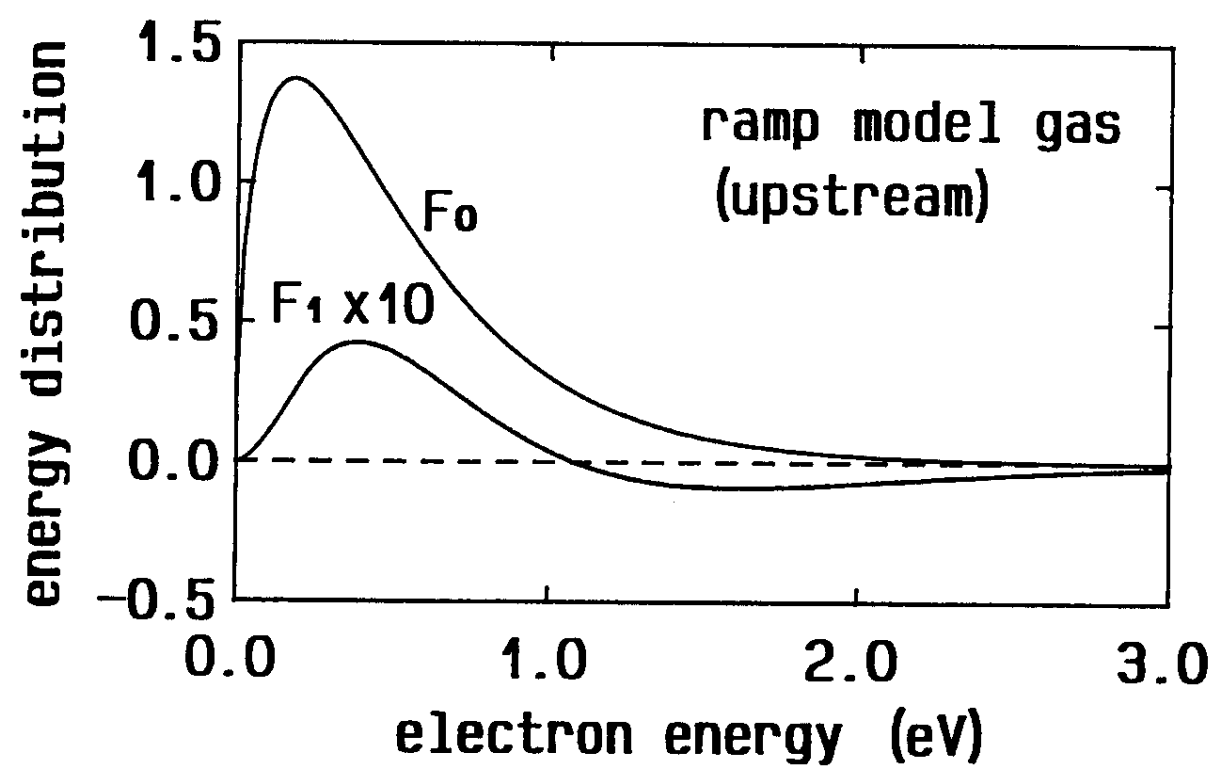

Figure 2(c)

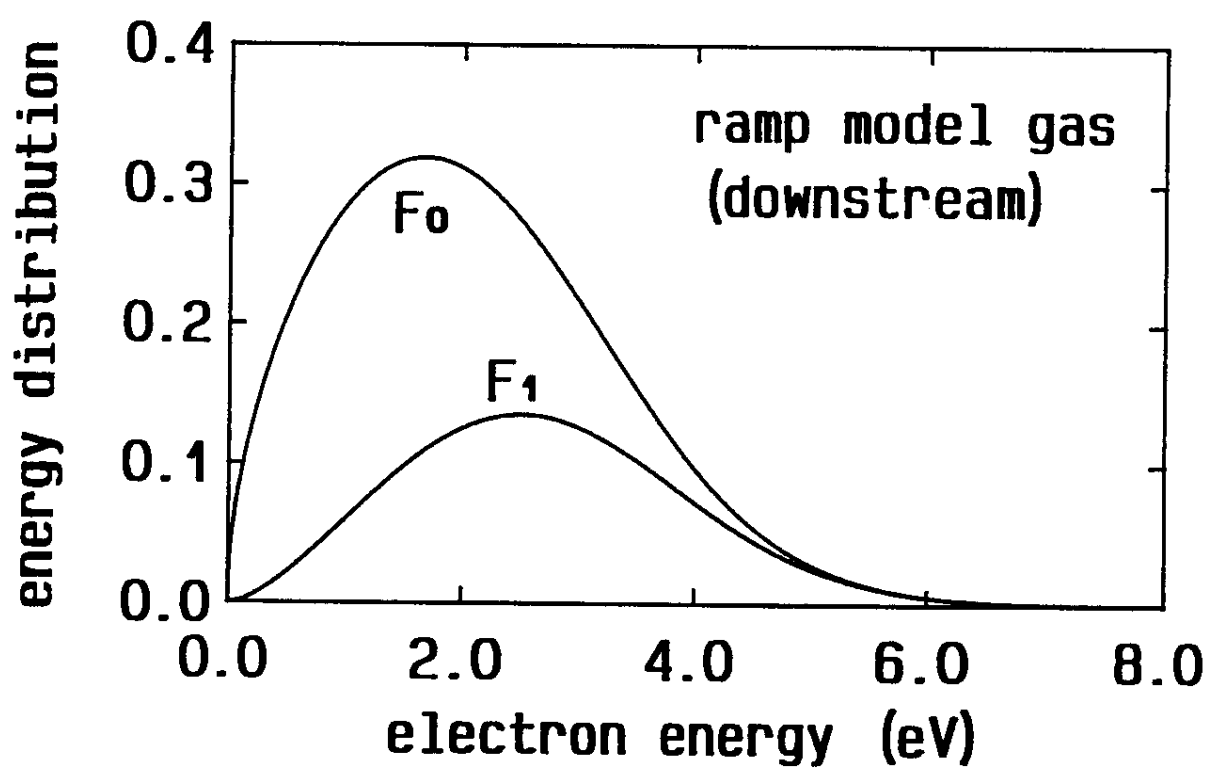

Figure 2(d)

Figure 2. Electron energy distributions calculated using a propagator technique: (a) and (b), argon at $E / N=$ $1410 \mathrm{Td}$; (c) and (d), ramp model gas at $E / N=283 \mathrm{Td}$; (e) and (f), sulphur hexafluoride at $E / N=141 \mathrm{Td}$. Figures (a), (c) and (e) are for the upstream region, while figures (b), (d) and (f) are for the downstream region. 


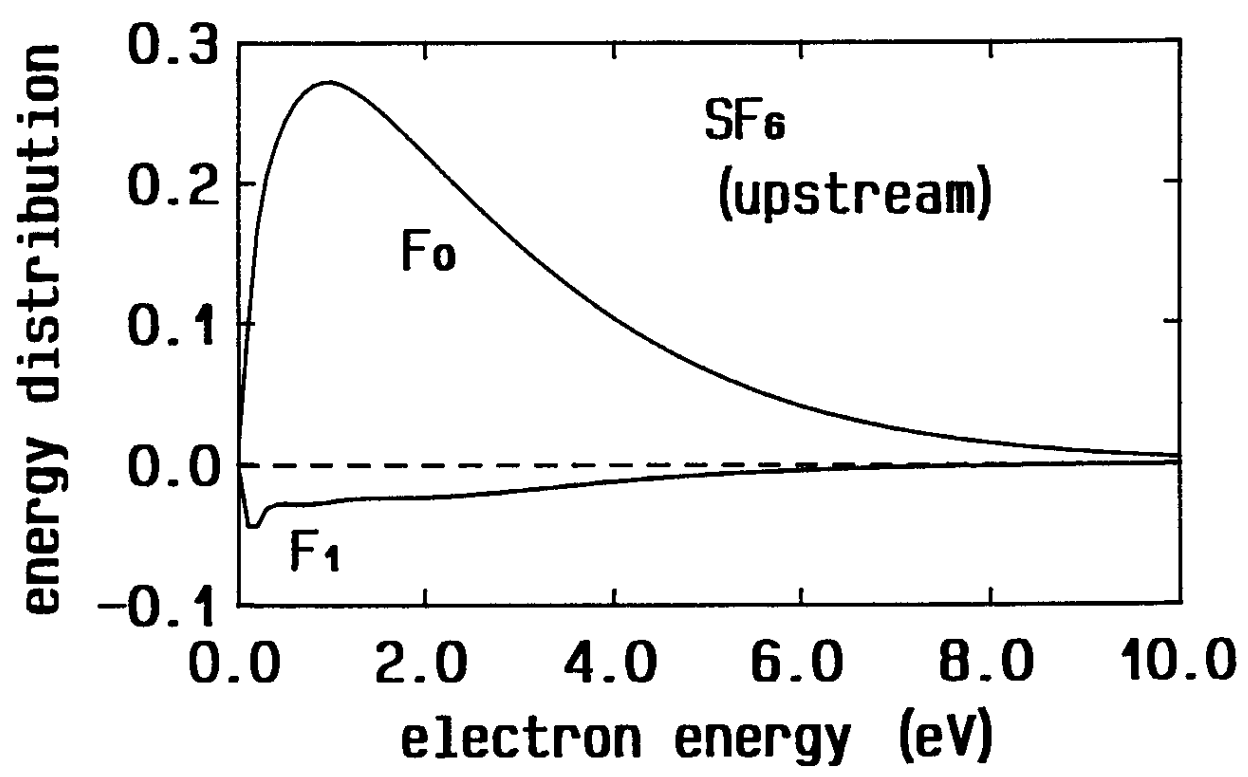

Figure 2(e)

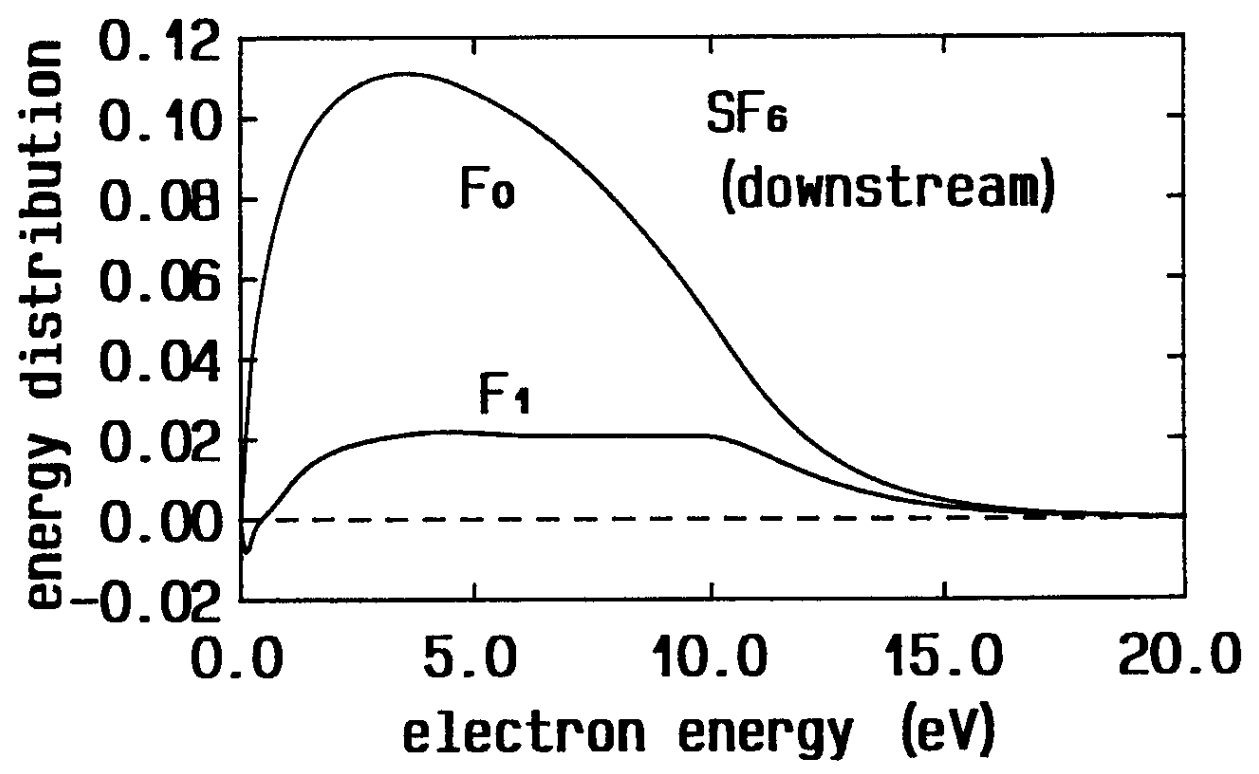

Figure 2(f)

Figure 2. Electron energy distributions calculated using a propagator technique: (a) and (b), argon at $E / N=$ $1410 \mathrm{Td}$; (c) and (d), ramp model gas at $E / N=283 \mathrm{Td}$; (e) and (f), sulphur hexafluoride at $E / N=141 \mathrm{Td}$. Figures (a), (c) and (e) are for the upstream region, while figures (b), (d) and (f) are for the downstream region. 


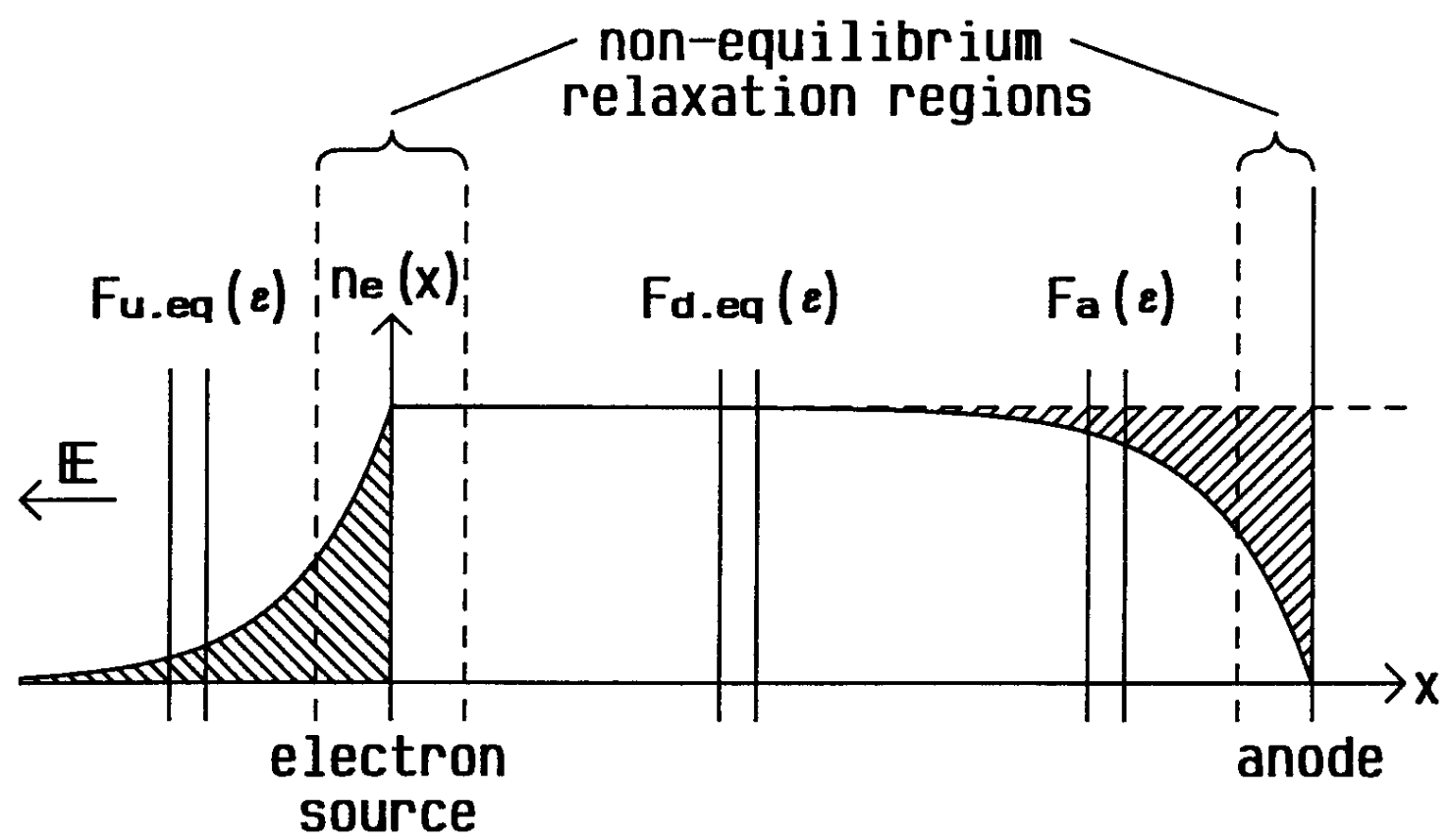

Figure 3. The model of a steady-state electron swarm in front of the anode. $F_{\text {u.eq }}(\varepsilon), F_{\text {d.eq }}(\varepsilon)$ and $F_{\mathrm{a}}(\varepsilon)$ represent the equilibrium electron energy distributions in the upstream and downstream regions and that under the influence of an absorbing anode respectively. The hatched part in front of the anode, given as $F_{\mathrm{v}}(\varepsilon, x)=F_{\text {d.eq }}(\varepsilon)-F_{\mathrm{a}}(\varepsilon, x)$, describes the distribution of missing electrons due to absorption. 


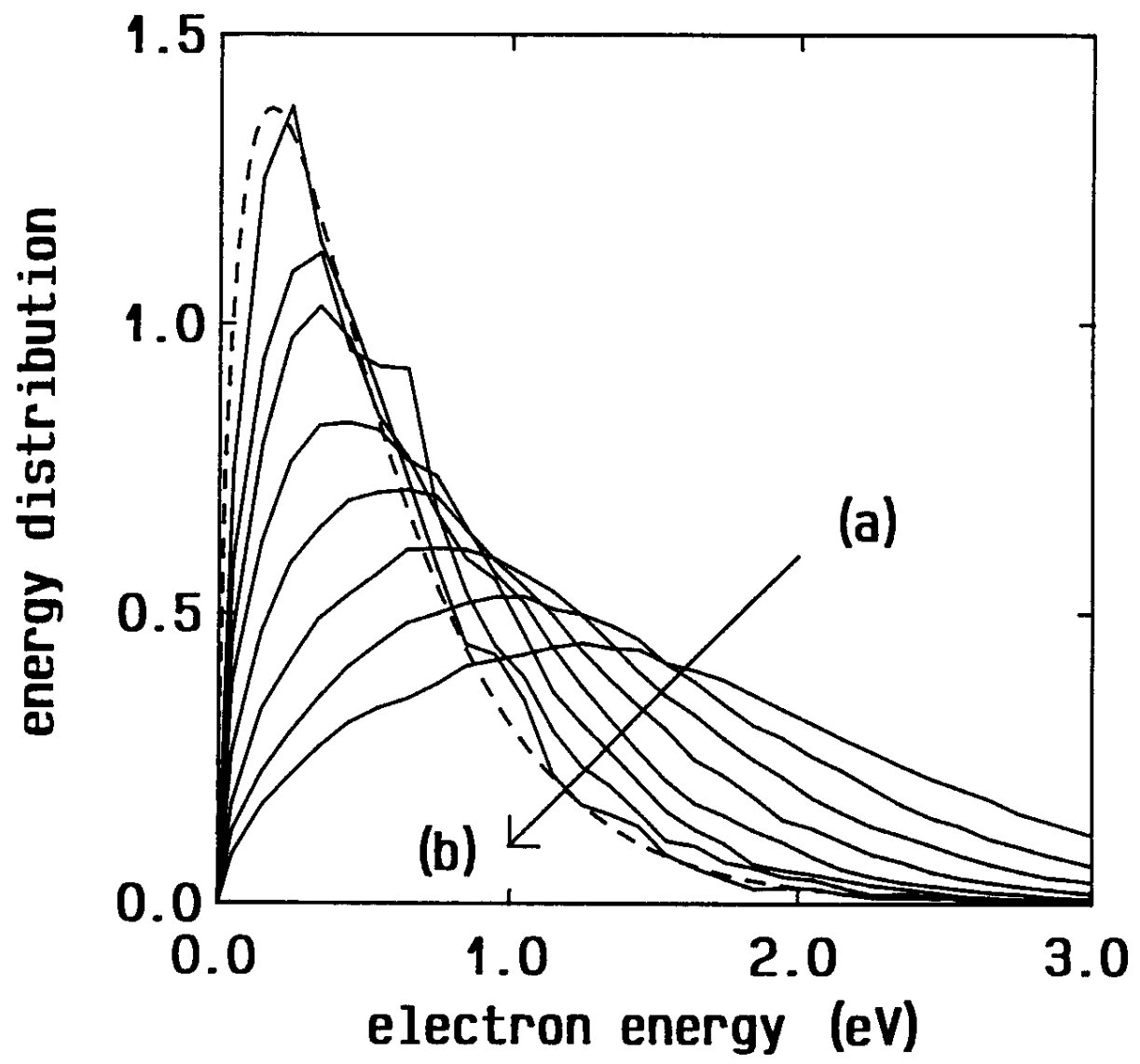

Figure 4. The energy distribution of backward-diffusing electrons in a ramp model gas obtained by a Monte Carlo simulation at $E / N=283 \mathrm{Td}$ as a function of distance from the anode. The electron energy distributions are presented for every $0.004 \mathrm{~cm}$ from (a) $-0.002 \mathrm{~cm}$ to (b) $-0.030 \mathrm{~cm}$. The electrons are assumed to move even after arrival at the anode. The distribution tends to the equilibrium solution indicated by the broken line, which is obtained by the present propagator method. 


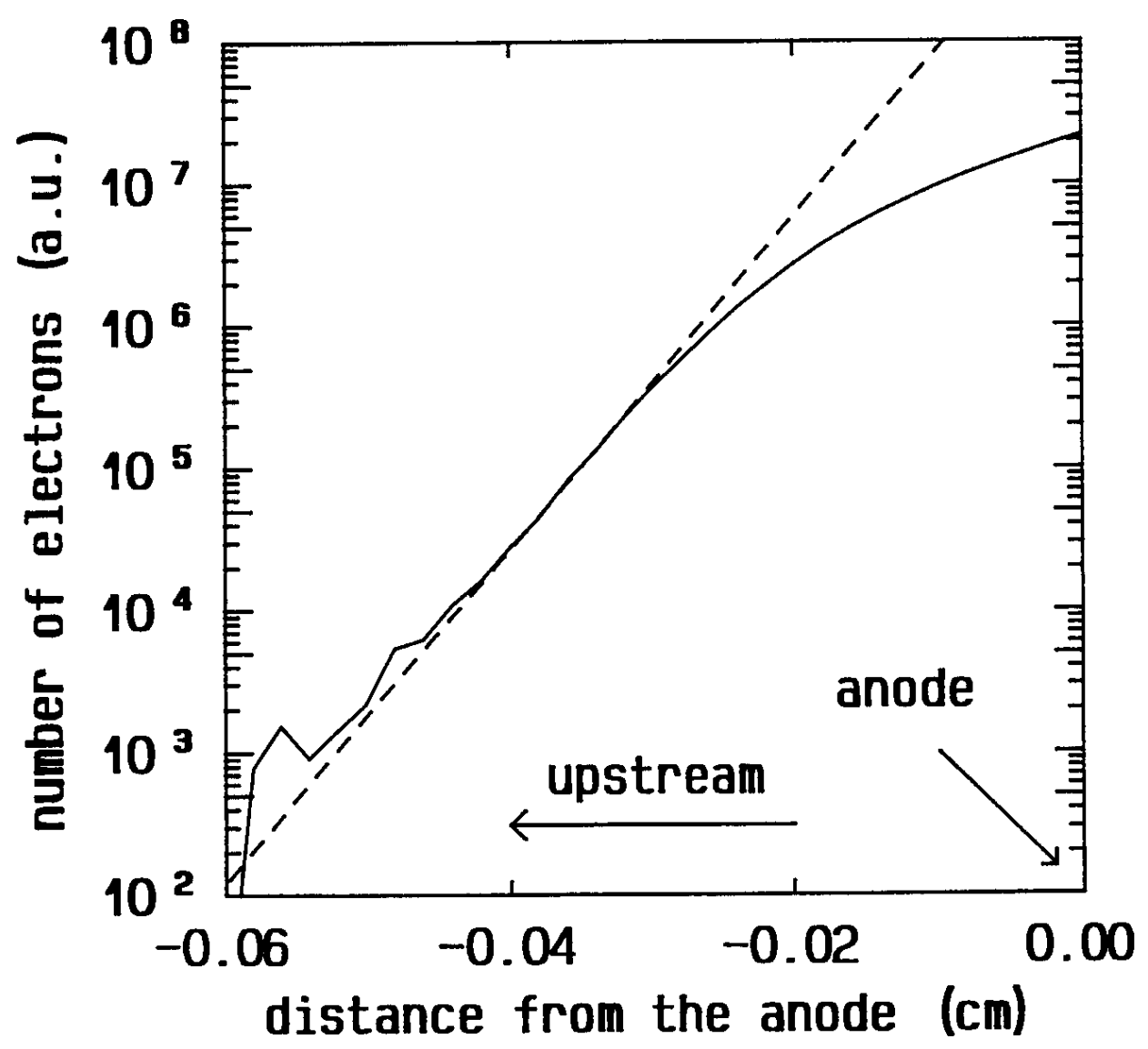

Figure 5. The number density of backward-diffusing electrons in a ramp model gas as a function of the distance from the absorbing anode under a steady-state Townsend condition obtained by a Monte Carlo simulation at $E / N=283 \mathrm{Td}$. Each electron is sampled just after its arrival at the anode. The broken line represents the relative density gradient, $\alpha$, from table 1 obtained by the present propagator method. The deviation from linearity towards the cathode is due to the low electron number density. 